\title{
Caracterización de mujeres embarazadas con lupus eritematoso sistémico y resultados materno-fetales en el noroeste de Colombia. Estudio descriptivo
}

Sara Manuela Ocampo-Ramirez', Simón Hoyos-Patiño ${ }^{1}$, José Raúl Lambertino-Montaño $0^{2,3}$, Jorge Hernán Gutiérrez-Marín ${ }^{4,5}$, María Nazareth Campo-Campo ${ }^{4,5}$, José Enrique Sanín-Blair ${ }^{4,5}$, Raúl Alejandro García-Posadaa ${ }^{4,5}$, Libia María Rodríguez-Padilla ${ }^{5}$, Carlos Jaime Velásquez-Franco ${ }^{5,6}$, Miguel Antonio Mesa-Nava5, ${ }^{5,6}$

\section{RESUMEN}

Objetivo: evaluar las características epidemiológicas, clínicas y de tratamiento de las mujeres embarazadas con lupus eritematoso sistémico, así como sus resultados obstétricos, en dos centros de referencia en el noroeste de Colombia.

Métodos: serie de casos retrospectiva que evaluó las gestantes atenđidas en la Unidad de Medicina Materno Fetal y en el servicio de Reumatología entre 2010 y 2015.

Resultados: se incluyeron 40 pacientes y 43 embarazos. Edad promedio al diagnóstico: 24 \pm 7 años; 22 (55 \%) fueron primigestantes. La afectación clínica más frecuente fue articular ( $\mathrm{n}=32 ; 80 \%$ ). Ocho de 43 embarazos (18,6\%) presentaron actividad lúpica al inicio de éste. La complicación obstétrica más frecuente fue preeclampsia en 11/43 (25,6 \%) embarazos. La complicación fetal más común fue el parto prematuro (8/43; 18,6\%). Los anticuerpos anticardiolipinas y anti-DNA fueron positivos en 10/43 (23,3\%) y en 15/38 (39,5\%) embarazos, respectivamente. En 13 gestaciones (31,7 \%) hubo recaídas durante el tercer trimestre, y de éstas, en seis casos (46,1\%) fue grave, especialmente nefritis. Al ingreso, en 29/43 (65\%) embarazos se utilizó prednisona y en 32/43 (74,4 \%) antimaláricos. En el puerperio dos pacientes presentaron recaída moderada y dos madres tuvieron preeclampsia. No se encontraron muertes maternas y la supervivencia perinatal fue $86 \%$ (37/43 gestaciones).

Discusión: en esta cohorte de pacientes con LES y embarazo, la nefritis y los anticuerpos antifosfolípidos estaban presentes en gran parte de las pacientes con complicaciones obstétricas,

1 Estudiantes Escuela de Ciencias de la Salud, Universidad Pontifica Bolivariana, Medellín, Colombia.

2 Especialista en Medicina Materno Fetal, Clínica SOMER, Rionegro, Colombia.

3 Especialista en Medicina Materno Fetal, Clínica del Prado, Medellín, Colombia.

4 Especialista en Medicina Materno Fetal, Clínica Universitaria Bolivariana, Medellín, Colombia.

5 Profesor Escuela de Ciencias de la Salud, Universidad Pontificia Bolivariana, Medellín, Colombia.

6 Reumatólogo, Clínica Universitaria Bolivariana, Medellín, Colombia.

Correspondencia: Sara Manuela 0campo-Ramírez; sara.ocampo@upb.edu.co

Recibido: octubre 17 de 2018

Aceptado: enero 31 de 2019

Cómo citar: Ocampo-Ramirez SM, Hoyos-Patiño S, Lambertino-Montaño JR, Gutiérrez-Marín JH, Campo-Campo MN, Sanín-Blair JE, et al. Caracterización de mujeres embarazadas con lupus eritematoso sistémico y resultados materno-fetales en el noroeste de Colombia. Estudio descriptivo. latreia. 2019 0ct-Dic;32(4):266-275. D0l 10.17533/udea.iatreia.25. 
aunque un bajo porcentaje de pacientes estuvo activa antes de la gestación; las complicaciones maternas y fetales fueron frecuentes.

\section{PALABRAS CLAVE}

Embarazo; Lupus Eritematoso Sistémico; Resultado del Embarazo

\section{SUMMARY}

Characterization of pregnant women with systemic lupus erythematosus and maternofetal outcomes in Northwestern Colombia: A descriptive study

Objective: To evaluate the epidemiological, clinical and therapeutic characteristics of pregnant women with systemic lupus erythematosus, as well as their obstetric results, in two reference centers in northwestern Colombia.

Methods: Retrospective case series that evaluated the pregnant women seen in the Maternal-Fetal Medicine and Rheumatology Units between 2010 and 2015.

Results: 40 patients and 43 pregnancies were included. The average age at diagnosis: $24 \pm 7$ years; 22 $(55 \%)$ were primigravidae. The most common clinical affectation was articular $(\mathrm{n}=32,80 \%)$. Eight of 43 pregnancies $(18.6 \%)$ presented lupus activity at the beginning of gestation. The most frequent obstetric complication was preeclampsia in $11 / 43$ (25.6\%) pregnancies. The most common fetal complication was preterm labor $(8 / 43,18.6 \%)$. Anticardiolipin and anti-DNA antibodies were positive in 10/43 (23.3\%) and in $15 / 38(39.5 \%)$ pregnancies, respectively. In 13 gestations $(31.7 \%)$, there were relapses during the third trimester, and of these, in six cases (46.1\%) there were severe especially nephritis. On admission, prednisone was used in 29/43 (65\%) pregnancies and antimalarial drugs in $32 / 43$ (74.4\%). In the puerperium, two patients had a moderate relapse and two mothers had preeclampsia. No maternal deaths were found and perinatal survival was $86 \%$ (37/43 pregnancies).

Discussion: In this cohort of patients with systemic lupus erythematosus and pregnancy, nephritis and antiphospholipid antibodies were present in a large part of the patients with obstetric complications, although a low percentage of patients were active before gestation; maternal and fetal complications were frequent.

\section{KEY WORDS}

Lupus Erythematosus, Systemic; Pregnancy; Pregnancy Outcome

\section{INTRODUCCIÓN}

El lupus eritematoso sistémico (LES) es una enfermedad autoinmune que puede afectar cualquier órgano o sistema y se caracteriza por un curso recurrente, con episodios de actividad y daño orgánico acumulado ${ }^{(1)}$. Afecta principalmente a las mujeres en edad reproductiva, con una relación estimada de nueve mujeres por cada hombre. Su incidencia anual aproximada es de 1,8-7,6/100.000 personas, con una prevalencia de $14,650,8 / 100.000$ personas, por lo que es una enfermedad relativamente rara ${ }^{(2)}$. No obstante, estas cifras presentan variaciones que dependen del área geográfica así como de la raza, que además predicen un compromiso más agresivo y un peor pronóstico ${ }^{(3)}$.

EI LES, en general, no compromete la fertilidad de las pacientes, pero puede afectar el desarrollo normal del embarazo y la salud del binomio madre-hijo ${ }^{(4,5)}$. Antes de 1950 se creía que los resultados obstétricos adversos en gestantes con LES sobrepasaban los favorables $y$, por tanto, se desaconsejaba embarazarse. Sin embargo, se ha observado una mejoría en el pronóstico general y, en consecuencia, en las tasas de éxito de los embarazos en mujeres con esta enfermedad ${ }^{(6.7)}$, probablemente por la introducción de nuevas terapias $y$ el conocimiento proveniente de distintas cohortes $y$ series de casos ${ }^{(4,6-9)}$, así como los seguimientos clínicos más estrechos y especializados.

En Latinoamérica la información disponible es escasa y proviene principalmente de series de casos de Chile, Aroentina, Brasil y México ${ }^{(10-14)}$, en las que se observa un comportamiento heterogéneo en cuanto a los desenlaces maternos, obstétricos y la actividad del lupus. Debido a esta variabilidad en los resultados y la falta de información local, se propuso evaluar las características epidemiológicas, clínicas y de tratamiento de las gestantes con LES y sus desenlaces obstétricos en dos centros de referencia en el noroeste de Colombia. 


\section{METODOLOGÍA}

\section{Diseño del estudio}

Se realizó un estudio descriptivo retrospectivo cuya población de estudio fueron las gestantes con diagnóstico de LES, atendidas tanto en la Unidad de Medicina Materno Fetal como en el servicio de Reumatología de dos hospitales de alto nivel de complejidad, desde enero de 2010 a diciembre de 2015. Se incluyeron las pacientes con diagnóstico confirmado de embarazo por ecografía y de LES según los criterios del American College of Rheumatology (ACR), modificados en $1999^{(15)}$. Se excluyeron las gestantes con otras enfermedades reumatológicas diferentes al SAF, diagnóstico de trombofilias hereditarias o información incompleta en la historia clínica del seguimiento del embarazo. Se indagó sobre las malformaciones congénitas (bloqueo cardíaco congénito) y no se turo en cuenta la etiología del hipotiroidismo.

\section{Proceso de recolección de la información}

Posterior al aval de los comités de ética de ambas instituciones, se procedió a revisar las historias clínicas identificadas con los códigos de la Clasificación Internacional de Enfermedades (CIE-10) relacionados: lupus y embarazo (M321, M328, M329, Z359, O800, O829). Posteriormente, se evaluó el cumplimiento de los criterios de elegibilidad y se diligenció un formato de recolección de información diseñado en la herramienta Magpi (disponible en https://home.magpi.com).

La información de las variables se obtuvo a partir de la historia clínica del control prenatal en alto riesgo obstétrico: hospitalización, parto o puerperio inmediato (hasta seis semanas posparto). (Tabla 1). Antes de la recolección de datos se realizó una prueba piloto en cinco registros clínicos que permitió modificar el instrumento antes de la recolección de la totalidad de los datos.

La información recolectada se exportó a Microsoft Excel (versión Office 2016), donde se hizo un análisis exploratorio que permitió evaluar la calidad de la información (datos faltantes, valores extremos, etc.) y la consistencia de los datos.

El sesgo de selección de la población se trató de controlar al incluir toda la población de pacientes que cumplió con los criterios de elegibilidad del estudio. Sin embargo, se trata de pacientes atendidos en centros de alta complejidad, lo que limita la aplicabilidad de los resultados. En cuanto al sesgo de información, con el fin de disminuir los errores, se diseñó una herramienta de recolección única, teniendo en cuenta los criterios de elegibilidad y las variables requeridas para esta investigación; se verificaron los datos que no coincidían con una revisión nueva de las historias clínicas por parte de uno de los investigadores.

Tabla 1. Variables incluidas en el proceso de recolección de datos

\begin{tabular}{|c|c|}
\hline Variable & \\
\hline Sociodemográficas & Edad, procedencia \\
\hline Antecedentes de LES & Compromiso articular, respiratorio, cutáneo, cardiaco y sistema nervioso central \\
\hline Antecedentes obstétricos & $\begin{array}{l}\text { Número de gestaciones, cesáreas, abortos, hijos vivos, mortinatos, parto prematuro, preeclampsia/ } \\
\text { eclampsia, insuficiencia placentaria definida como restricción del crecimiento intrauterino (RCIU)* }\end{array}$ \\
\hline Comorbilidades maternas & Hipertensión arterial, diabetes mellitus, dislipidemia, enfermedad tiroidea \\
\hline Variables de laboratorio & $\begin{array}{l}\text { Presencia de anticuerpos antinucleares (ANA), patrón de ANA, anti-DNA, complemento (C3 y C4), } \\
\text { proteínas en la orina de } 24 \text { horas, ENAS (Ro, La, Sm, anti-RNP), anticuerpos antifosfolípidost }\end{array}$ \\
\hline Complicaciones & $\begin{array}{l}\text { Aborto, óbito fetal, muerte materna, preeclampsia, eclampsia, RCIU, parto pretérmino, ruptura de } \\
\text { membranas, diabetes gestacional, sepsis o neumonía, actividad lúpicał }\end{array}$ \\
\hline Seis semanas posparto & Actividad lúpica, trombosis arterial, trombosis venosa profunda, embolia cerebral, hemorragia \\
\hline Tratamiento & $\begin{array}{l}\text { Uso de cloroquina, hidroxicloroquina, prednisolona, azatioprina, micofenolato mofetil, ciclofosfa- } \\
\text { mida, rituximab, gammaglobulina, tacrolimus, ciclosporina }\end{array}$ \\
\hline
\end{tabular}

* Peso fetal estimado menor del percentil 3 o menor al percentil 10 con alteración del Doppler, según las definiciones establecidas en la literatura (1618). †Anticoagulante lúpico, lgG e lgM para B2-glicoproteína y anticuerpos anticardiolipina (19). ‡Definida por el criterio del médico tratante o según los términos del Systemic Lupus Erithematosus Disease Activity Index [SLEDAI] (20). Fuente: elaboración propia 


\section{Análisis estadístico}

Para describir las variables cuantitativas se utilizó el promedio y la desviación estándar (DE) o la mediana y el rango intercuartílico (RIQ) según la distribución de los datos. Las variables categóricas se expresaron en frecuencias absolutas $y$ proporciones. Todos los análisis se llevaron a cabo en IBM SPSS 22.

\section{RESULTADOS}

\section{Características sociodemográficas y clínicas al ingreso}

Se revisaron 2139 historias clínicas, de estas, 1998 pacientes fueron excluidas por solo presentar LES o por estar en embarazo sin LES, resultando 47 pacientes que cumplían con los criterios de inclusión, de las cuales siete se excluyeron por tener datos incompletos para el seguimiento, quedando 40 pacientes con un total de 43 embarazos. La edad promedio al momento del diagnóstico de LES fue $24 \pm 7$ años. La mayoría de las pacientes estaban afiliadas al régimen de seguridad en salud 38/40 (95\%) y, 40/43 (93\%) no planearon la gestación. Las principales manifestaciones lúpicas en este grupo de pacientes fueron las articulares $32 / 40(80 \%)$, seguido por las mucocutáneas 26/40 (65 \%), hematológicas 16/40 (40\%) y renales 13/40 (32\%) (Tabla 2).

La información de la clase histológica de nefritis lúpica estaba disponible en nueve de trece pacientes, de estas 6/9 (66 \%) eran nefritis clase IV y $3 / 9$ (33\%) nefritis clase V. Respecto a la actividad lúpica, solo 8/43 (18,6 \%) embarazos presentaban actividad lúpica al ingreso al estudio.

Ninguna paciente turo antecedente de trombosis arterial o venosa, ni relató el consumo de sustancias como el alcohol o cigarrillo. Con respecto al LES, solo en un embarazo (2,5\%), el diagnóstico se realizó durante el período gestacional.

\section{Características obstétricas al ingreso}

Veintidós $(55 \%)$ de las pacientes eran primigestantes. El número de embarazos previos en las pacientes multíparas fue $21, y$ de estas, el antecedente de pérdida gestacional fue el más frecuente 15/21 (71,4 $\%)$; donde en 13 de estos casos (86,7\%) ocurrió en el embarazo previo al del ingreso al estudio. Los antecedentes de insuficiencia placentaria y parto prematuro ocurrieron en la misma frecuencia $3 / 21(14,3 \%)$, y en 2/21 casos $(9,5 \%)$ se presentaron mortinatos.
Tabla 2. Características sociodemográficas y clínicas de las gestantes con lupus eritematoso sistémico $(n=40)$

\begin{tabular}{lc}
\hline \multicolumn{1}{c}{ Característica } & $\mathbf{n}(\%)$ \\
$\begin{array}{l}\text { Edad al diagnóstico de LES (años)* } \\
\mathrm{n}=37\end{array}$ & $24 \pm 7(7-38)$ \\
$\begin{array}{l}\text { Procedencia }(\mathrm{n}=37) \\
\quad \text { Urbana }\end{array}$ & $35(94,3)$ \\
$\quad$ Rural & $2(5,7)$ \\
Régimen de salud & \\
Contributivo & $36(90)$ \\
Subsidiado & $2(5)$ \\
Sin afiliación & $2(5)$ \\
Comorbilidades & \\
Enfermedad tiroidea & $13(32,5)$ \\
Hipertensión & $5(12,5)$ \\
Dislipidemia & $3(7,5)$ \\
Compromiso lúpico & \\
Articular & $32(80)$ \\
Mucocutáneo & $26(65)$ \\
Hematológico & $16(40)$ \\
Nefritis & $13(32)$ \\
Sistema nervioso central & $12(30)$ \\
Respiratorio & $4(10)$ \\
Cardíaco & $3(7,5)$ \\
\hline
\end{tabular}

* Media \pm DE (rango). Fuente: elaboración propia

En 14/36 embarazos (39 \%) había antecedente del síndrome antifosfolípido (SAF). De los que se tenía información 2/5 (40\%) fueron trombóticos y 3/5 (60\%) obstétricos. Ninguna paciente presentó antecedente de preeclampsia (Tabla 3).

Tabla 3. Antecedentes obstétricos en una cohorte de lupus eritematoso sistémico y embarazo

\begin{tabular}{lc}
\hline \multicolumn{1}{c}{ Antecedente } & $\mathbf{n} / \mathbf{N}(\%)$ \\
Insuficiencia placentaria & $3 / 40(7,5)$ \\
\hline Pérdida gestacional & $15 / 40(37,5)$ \\
1 & $13 / 15(86,7)$ \\
2 & $1 / 15(6,7)$ \\
3 & $1 / 15(6,7)$ \\
\hline Cesáreas & $13 / 40(32,5)$ \\
Número de gestaciones & \\
1 & $22 / 40(55,0)$ \\
2 & $16 / 40(40,0)$ \\
Más de 2 & $2 / 40(5,0)$ \\
Número de mortinatos* & $2 / 21(9,5)$ \\
Partos prematuros* & $3 / 21(14)$ \\
\hline
\end{tabular}

* Estimado del total de embarazos previos. Fuente: elaboración propia 


\section{Características de laboratorio al ingreso}

Los resultados de ANA fueron positivos en 25/27 de las pacientes $(92,6 \%)$, siendo el patrón moteado el más frecuente 12/17 (70,6 \%). Los anticuerpos anti-Ro y anti-La fueron positivos en 19/38 (50 \%) y, 12/38 (31,6\%) de las mujeres, en ninguno de los casos se presentó un bloqueo cardíaco congénito. Con respecto al perfil de los anticuerpos antifosfolípidos, 2/43 embarazos (4,6\%) presentaron triple positividad y uno de estos casos cursó con óbito fetal durante un embarazo previo. Los anticuerpos anticardiolipina fueron positivos en 10/43 (23,3 \%) embarazos (Tabla 4), de estos, $3 / 10$ (30 \%) casos tenían antecedentes de aborto en gestaciones previas, 2/10 (20 $\%)$ presentaron preeclampsia y 1/10 (10 \%) RCUI.

Durante el seguimiento de la gestación estas pacientes desarrollaron complicaciones relacionadas como preeclampsia $3 / 10(30 \%)$, RCIU 2/10 (20\%) y parto pretérmino 2/10 (20\%). En 11/21 (42\%) embarazos se encontró proteinuria mayor a $300 \mathrm{mg}$, en ocho la proteinuria fue mayor a $500 \mathrm{mg} y$, en dos mayor a 3,5 gramos.

En cuatro de las ocho pacientes con proteinuria mayor a $500 \mathrm{mg}$ y en las dos que presentaron proteinuria mayor a 3,5 gramos al día esto fue atribuido a la nefritis lúpica. Con respecto a la creatinina, en cuatro casos se presentaron valores por encima del rango de referencia para la edad gestacional y en todos estos hubo presencia de nefritis activa.

\section{Tratamiento}

Al ingreso del control prenatal 29/43 (65 \%) embarazos utilizaron prednisona (dosis mediana: $10 \mathrm{mg}$ (RIQ: 5-15) y en 32/43 (74,4 \%) antimalárico, en 21/32 (65,6 \%) de Ios casos se usó cloroquina y, en 11/32 (34,4 \%) hidroxicloroquina. El único ahorrador de esteroides utilizado fue la azatioprina 14/43 (32,6 \%). No hubo exposición a la ciclofosfamida en el embarazo, se desconoce si en períodos anteriores las pacientes estuvieron expuestas.

Durante el curso de los embarazos en $36 / 43$ casos (83,7 $\%$ ) se administró prednisona (n: 33 dosis mediana: 10; RIQ: 8 -20), teniendo un aumento del $22,7 \%$ en el uso de este medicamento (65\% al ingreso y $83,7 \%$ durante el seguimiento). En 15/43 (35\%) embarazos se utilizó aspirina. Solo en dos (5\%) se suspendió el antimalárico y, si bien no se reactivó la enfermedad de base, sí se presentaron complicaciones en ambos casos, específicamente preeclampsia y parto pretérmino.
Tabla 4. Hallazgos del perfil autoinmune en una cohorte de lupus eritematoso sistémico y embarazo

\begin{tabular}{lc}
\hline \multicolumn{1}{c}{ Hallazgo de laboratorio } & $\mathbf{n} / \mathbf{N \dagger}(\%)$ \\
\hline ANA positivos & $25 / 27(92,6)$ \\
\hline $\begin{array}{l}\text { Patrón ANA* } \\
\text { Moteado }\end{array}$ & $12 / 17(70,6)$ \\
Homogéneo & $5 / 17(29,4)$ \\
\hline Anti-DNA & $15 / 38(39)$ \\
\hline Anti-Ro & $19 / 38(50)$ \\
\hline Anti-La & $12 / 38(31,6)$ \\
\hline Anti-Sm & $16 / 39(41,0)$ \\
\hline Anti-RNP & $19 / 38(50)$ \\
\hline Anticoagulante lúpico & $8 / 43(18,6)$ \\
\hline Anti-B2 glicoproteína 1 IgG & $4 / 43(9,3)$ \\
\hline Anti-B2 glicoproteína 1 IgM & $4 / 43(9,3)$ \\
\hline Anticuerpos anticardiolipina & $10 / 43(23,3)$ \\
\hline IgG & $3 / 10$ \\
\hline IgM & $6 / 10$ \\
\hline Hipocomplementemia C3 & $13 / 43(30)$ \\
\hline Hipocomplementemia C4 & $11 / 43(25)$ \\
\hline * ANA: Anticuerpos antinucleares & \\
+ Los cálculos se realizaron de acuerdo al total de embarazos con datos \\
disponibles. Fuente: elaboración propia
\end{tabular}

\section{Complicaciones y desenlaces}

Con respecto a las complicaciones derivadas del LES, se presentaron siognos de reactivación en 13/41 (31,7\%) embarazos, siendo las manifestaciones renales las observadas con mayor frecuencia $7 / 43$ (16,3\%). En 6/13 casos $(46,1 \%)$ la reactivación se clasificó como grave por presentar trombocitopenia amenazante de la vida, o glomerulonefritis rápidamente progresiva. Otras complicaciones fueron la diabetes gestacional 3/43 (7 \%) y la sepsis urinaria 2/43 (4,7 \%) (Tabla 5), un caso en la semana 29 y el otro en la semana 11 , respectivamente; el primero complicado con el parto pretérmino y el último terminó con un aborto. Desde el punto de vista obstétrico no se encontraron muertes maternas y la supervivencia perinatal fue del $86 \%$, pues en $3 / 43$ ( $7 \%$ ) casos se presentó aborto espontáneo y, 3/43 (7\%) muertes fetales; una de estas pacientes cursaba con SAF obstétrico. La preeclampsia fue la complicación más frecuente en 11/43 (25,6 \%) casos, presentándose en dos oportunidades en el segundo trimestre, en las semanas 20 y 26; en ningún caso se complicó con eclampsia. 
Con relación a la preeclampsia y la nefritis lúpica, se observó la primera en 6/13 (46,1 \%) de los embarazos con nefritis, mientras que, en los embarazos de las pacientes sin nefritis, la preeclampsia ocurrió en 5/30 casos $(16,7 \%)$. No se presentaron muertes maternas durante el tiempo del estudio. Las complicaciones fetales más comunes fueron el parto prematuro $8 / 43$ $(18,6 \%)$ y la restricción del crecimiento intrauterino 6/43 (14\%) (Tabla 5).

\section{Tabla 5. Complicaciones y desenlaces obstétricos en una cohorte de lupus eritematoso sistémico y embarazo $(n=43)$}

\begin{tabular}{lc}
\hline \multicolumn{1}{c}{ Complicaciones y desenlaces } & $\mathbf{n} / \mathbf{N}(\%)$ \\
$\begin{array}{l}\text { Compromiso de órgano } \\
\text { Piel }\end{array}$ & $6(14,0)$ \\
Articular & $1(2,3)$ \\
$\quad$ Riñón & $7(16,3)$ \\
\hline Reactivación lúpica & $13 / 41(31,7)$ \\
$\quad$ Leve & $4 / 13(30,8)$ \\
Moderada & $3 / 13(23,1)$ \\
Grave & $6 / 13(46,1)$ \\
Diabetes gestacional & $3(7,0)$ \\
Sepsis & $2(4,7)$ \\
RCIU* & $6(14,0)$ \\
Parto prematuro & $8(18,6)$ \\
Hemorragia posparto & $1(2,3)$ \\
Ruptura de las membranas & $7(16,3)$ \\
\hline Preeclampsia & $11(25,6)$ \\
\hline Aborto & $3(7,0)$ \\
\hline Óbito fetal & $3(7,0)$ \\
\hline
\end{tabular}

* RCIU: Restricción del crecimiento intrauterino. Fuente: elaboración propia

En el puerperio, dos pacientes tuvieron una recaída lúpica moderada (nefritis) y dos madres con preeclampsia requirieron de un ajuste de los fármacos antihipertensivos. En este período no se documentaron malformaciones congénitas.

\section{DISCUSIÓN}

Estudios previos de pacientes con LES y embarazo en otras poblaciones han informado que existen más probabilidades de presentar afección articular, cutánea y renal, así como reactivación de la enfermedad y desenlaces adversos, tanto maternos como fetales ${ }^{(21-}$ ${ }^{23}$. De esta cohorte se destaca que fueron comunes los desenlaces adversos obstétricos en las pacientes con anticuerpos antifosfolípidos positivos ${ }^{(10)}$ y que fue más frecuente la presencia de los trastornos hipertensivos asociados al embarazo, así como los desenlaces adversos en los pacientes con antecedente de nefritis $(5,9,21,24)$. Hasta donde se sabe, este es el primer estudio que provee información local sobre el tema.

En nuestra población, la mayoría de las pacientes estaban en la tercera década de la vida, igual a lo reportado por otras series latinoamericanas ${ }^{(10-14,25)}$. Sin embargo, el porcentaje de hipertensión crónica fue mayor a lo descrito en otros estudios ${ }^{(5,9)}$, probablemente, dado el mayor número de pacientes con compromiso renal previo.

En cuanto a la afección lúpica al ingreso, las principales manifestaciones fueron articulares $y$ mucocutáneas, resultados similares a otros estudios ${ }^{(9,24,26)}$; aclaramos que la mayoría de las pacientes estaban en remisión al momento del ingreso a la cohorte. Durante el seguimiento las manifestaciones renales y cutáneas fueron las más frecuentes, similar a lo descrito por Petri et al, ${ }^{(27)}$, en una cohorte de 74 embarazos con pacientes de raza mixta, en la cual predominaron estas manifestaciones, además del compromiso articular, que en nuestra población no fue tan frecuente.

Respecto al compromiso renal, un tercio de las gestantes tenía nefritis al ingreso y, un $16 \%$ desarrolló un compromiso renal durante la gestación. También se observó una mayor frecuencia de los trastornos hipertensivos asociados al embarazo, a pesar de que los valores de creatinina permanecieron estables en más del $90 \%$ de las pacientes, esto es acorde a lo reportado en estudios previos ${ }^{(21,28)}$ y resalta el papel de la nefritis en los desenlaces obstétricos, no solo por la actividad misma sino por el daño renal crónico y sus consecuencias.

Con relación al perfil inmunológico, el patrón de los ANA más frecuente fue el moteado, lo que difiere de otras publicaciones donde se reporta el homogéneo como el más común ${ }^{(24)}$, explicado posiblemente por las variaciones en el reporte de estos. La positividad de los anticuerpos anti-Ro y anti-La fue similar a otros estudios ${ }^{(22)}$, explicando la baja frecuencia del compromiso neonatal. Las pacientes con anticuerpos antifosfolípidos no solo tenían antecedentes de resultados adversos obstétricos, sino que, en el transcurso 
de la gestación, el $60 \%$ de estos casos, turo un nuevo desenlace adverso, lo que concuerda con los datos de una población chilena ${ }^{(10)}$ y resalta la importancia de la coexistencia de estas dos enfermedades.

La frecuencia del uso de prednisolona durante el curso del embarazo tuvo un aumento del $22,7 \%$ con respecto al ingreso, en contraste con otro estudio, donde este incremento fue cercano al $5 \%{ }^{(29)}$, diferencia, probablemente explicada, por un protocolo de manejo distinto al de nuestra región. Sin embargo, el uso de prednisolona en dosis inferiores a $20 \mathrm{mg}$ al día, se mantuvo durante el curso de la gestación, dosis indicada en aqueIlas pacientes con recaída aguda no grave del LES ${ }^{(30)}$.

La mayoría de las pacientes fueron tratadas con antimálaricos, siendo el más frecuente la cloroquina; lo que pudiera ser explicado por la mayor disponibilidad de este medicamento en nuestro sistema de salud. Además, el porcentaje de uso de la hidroxicloroquina fue similar a otros estudios ${ }^{(31-32)}$. Cabe resaltar que, aunque la literatura muestra que la hidroxicloroquina es más segura durante el embarazo ${ }^{(33)}$, ninguna de las pacientes que recibió cloroquina presentó resultados adversos secundarios a este medicamento.

Es de mencionar que, en el presente estudio, solo hubo dos casos de abandono del antimalárico y en ambos se presentaron desenlaces obstétricos adversos, lo cual resalta la importancia de no suspender este medicamento ${ }^{(34-35)}$. Adicionalmente, un bajo porcentaje de embarazadas utilizó la aspirina como estrategia de prevención primaria para la preeclampsia, sin que se evidenciara un aumento en su frecuencia al compararla con otros estudios, pese al mayor porcentaje de pacientes con hipertensión crónica y compromiso renal preexistente en la presente serie, o por un inicio tardío de esta luego de la placentación ${ }^{(4-9,96)}$.

Si bien los datos del SLEDAI no estuvieron disponibles en toda la población, el 18,6 \% de las pacientes presentaban actividad al ingreso de acuerdo con el criterio de los médicos tratantes, valor que llegó hasta el 31,7 \% durante el embarazo, siendo la manifestación grave, la forma más frecuente de reactivación. Los resultados de los estudios al respecto no han sido concluyentes ${ }^{(5 .}$ 37-39), en parte, dado por la dificultad para evaluar algunos signos y los síntomas de reactivación de la enfermedad, los cuales se pueden observar en la población obstétrica como fenómenos fisiológicos o, algunas veces, patológicos relacionados con el embarazo, como rash malar, artralgias, preeclampsia, entre otros ${ }^{(40-41)}$. Sin embargo, estos datos están en concordancia con algunos de los estudios publicados previamente y refuerzan la importancia de iniciar la gestación con al menos seis meses de quiescencia de la enfermedad, como recomiendan distintos expertos en el tema ${ }^{(42-43)}$.

La preeclampsia fue la principal complicación observada en esta cohorte, y se encontró una similitud con los datos publicados por Chakravarty et al, (22\%) (44) y Clowse et al., $(22,5 \%)^{(5)}$. Aunque estas series están conformadas por poblaciones étnicas distintas, hay similitud en la edad promedio durante la gestación, el compromiso renal basal y otros factores como la trombocitopenia, los cuales han sido descritos como factores de riesgo para el desarrollo de trastornos hipertensivos del embarazo en LES ${ }^{(44)}$. Respecto a otras complicaciones, el aborto espontáneo y la muerte fetal se presentaron en nuestra cohorte con una proporción similar a lo ya descrito en otros casos ${ }^{(34,44-46)}$.

Por ser nuestro estudio retrospectivo, basado en la revisión de historias clínicas, una de sus principales limitaciones está dada por la posibilidad de errores en el almacenamiento y el registro de datos consiognados en la historia clínica. Sin embargo, se utilizaron varias estrategias para garantizar la calidad de los datos, como por ejemplo, revisar nuevamente las historias clínicas en caso de datos faltantes o discordantes. Por otra parte, al tratarse de dos instituciones de tercer nivel de carácter privado, la población en estudio pudiese no representar el universo de pacientes de la región, lo cual se ve reflejado en el tipo de aseguramiento de estas en la muestra. No obstante, cabe resaltar que, en conjunto los dos centros médicos atienden en promedio 15000 nacimientos anuales, lo que representa un gran porcentaje de los embarazos de la ciudad. Adicionalmente, si bien estos dos centros son de alta complejidad, dada las características propias de los embarazos con LES, es poco probable que estos se manejen en otros ámbitos. Aunque solo se lograron captar $43 \mathrm{em}-$ barazos, el período de reclutamiento fue amplio y el número evaluado es similar al reportado en cohortes internacionales y series latinoamericanas.

\section{CONCLUSIONES}

De esta cohorte de pacientes colombianas con lupus eritematoso sistémico y embarazo, se destaca la mayor 
frecuencia de los trastornos hipertensivos asociados al embarazo y los desenlaces adversos en pacientes con antecedente de nefritis. Los desenlaces obstétricos negativos fueron más frecuentes en los pacientes con la presencia de anticuerpos antifosfolípidos. La recaída lúpica fue frecuente y, en la mayoría de los casos, grave.

No se encontraron efectos adversos o mayor frecuencia de las complicaciones asociadas al uso de cloroquina durante la gestación.

Se espera que los resultados de este trabajo sirvan como punto de partida para el desarrollo de nuevos estudios y así ampliar el conocimiento en esta área, buscando un mejor manejo de este grupo de pacientes.

\section{CONFLICTOS DE INTERESES}

Ninguno por declarar.

\section{REFERENCIAS BIBLIOGRÁFICAS}

1. Bertsias G, Cervera R, Boumpas DT. Systemic lupus erythematosus: pathogenesis and clinical features [Internet]. [Consultado 2019 Mar 4]. Disponible en: https:// cutt.ly/twZo1wt.

2. Lim SS, Drenkard C. Epidemiology of lupus: an update. Curr Opin Rheumatol. 2015;27(5):427-32. DOI 10.1097/BOR.0000000000000198.

3. Shabanova SS, Ananieva LP, Alekberova ZS, Guzov II. Orarian function and disease activity in patients with systemic lupus erythematosus. Clin Exp Rheumatol. 2008 May-Jun;26(3):436-41.

4. Buyon JP, Kim MY, Salmon JE. Predictors of Pregnancy Outcomes in Patients with Lupus. Ann Intern Med. 2016;164(2):131. DOI 10.7326/L15-0500.

5. Clowse ME, Jamison M, Myers E, James AH. A national study of the complications of lupus in pregnancy. Am J Obstet Gynecol. 2008;199(2):127.e1-6. DOI 10.1016/j. ajog. 2008.03.012.

6. Al Arfaj AS, Khalil N. Pregnancy outcome in 396 pregnancies in patients with SLE in Saudi Arabia. Lupus. 2010;19(14):1665-73. DOI 10.1177/0961203310378669.

7. Ambrósio P, Lermann R, Cordeiro A, Borges A, Nogueira I, Serrano F. Lupus and pregnancy--15 years of experience in a tertiary center. Clin Rev Allergy Immunol. 2010;38(2-3):77-81. DOI 10.1007/s12016-009-8139-9.
8. Clark CA, Spitzer KA, Laskin CA. Decrease in pregnancy loss rates in patients with systemic lupus erythematosus over a 40-year period. J Rheumatol. 2005;32(9): 1709-12.

9. Schreiber K. Pregnancies in women with systemic lupus erythematosus and antiphospholipid antibodies. Lupus. 2016;25(4):343-5. DOI 10.1177/0961203315627201.

10. Sabat S, Vinet M, Sanhueza C, Galdames A, Castro I, Caballero A. Embarazadas con lupus eritematoso sistémico en el hospital Clínico Regional de Concepción. Chile Rev chil obstet ginecol. 2014;79(1) : 21-6. DOI 10.4067/S0717-75262014000100004.

11. Gutiérrez R, Caro J, Pérez L, Ramírez J. Lupus eritematoso sistemico y embarazo. Rev chil obstet ginecol. 2002; 67(2) : 121-4. DOI 10.4067/S0717-752620020002000007.

12. Cavallasca JA, Laborde HA, Ruda-Vega H, Nasswetter GG. Maternal and fetal outcomes of 72 pregnancies in Argentine patients with systemic lupus erythematosus (SLE). Clin Rheumatol. 2008;27(1):41-6. DOI 10.1007/s10067-007-0649-3.

13. Naseri EP, Surita FG, Borovac-Pinheiro A, Santos M, Appenzeller S, Costallat LTL. Systemic Lupus Erythematosus and Pregnancy: A Single-Center Observational Study of 69 Pregnancies. Rev Bras Ginecol Obstet. 2018;40(10):587-592. DOI 10.1055/s-0038-1672136.

14. Saavedra MÁ, Miranda-Hernández D, Sánchez A, Morales $\mathrm{S}$, Cruz-Domínguez $\mathrm{P}$, Medina $\mathrm{G}$ et al. Pregnancy outcomes in women with childhood-onset and adult-onset systemic lupus erythematosus: a comparative study. Rheumatol Int. 2016;36(10):1431-7. DOI 10.1007/s00296-016-3525-0.

15. Hochberg MC. Updating the American College of Rheumatology revised criteria for the classification of systemic lupus erythematosus. Arthritis Rheum. 1997;40(9):1725. DOI 10.1002/art.1780400928.

16. American College of Obstetricians and Gynecologists' Committee on Practice Bulletins-Obstetrics. Practice Bulletin No. 171: Management of Preterm Labor. Obstet Gynecol. 2016 Oct;128(4):e155-64. DOI 10.1097/AOG.0000000000001711.

17. Report of the National High Blood Pressure Education Program Working Group on High Blood Pressure in Pregnancy. Am J Obstet Gynecol. 2000;183(1):S1-S22.

18. American College of Obstetricians and Gynecologists, Task Force on Hypertension in Pregnancy. Hypertension in pregnancy. Report of the American College of 
Obstetricians and Gynecologists' Task Force on Hypertension in Pregnancy. Obstet Gynecol. 2013;122(5):112231. DOI 10.1097/01.AOG.0000437382.03963.88.

19. Tan EM, Cohen AS, Fries JF, Masi AT, McShane DJ, Rothfield NF, et al. The 1982 revised criteria for the classification of systemic lupus erythematosus. Arthritis Rheum. 1982 Nov;25(11):1271-7.

20. Gladman DD, Goldsmith CH, Urowitz MB, Bacon P, Bombardier $C$, Isenberg $D$. Sensitivity to change of 3 Systemic Lupus Erythematosus Disease Activity Indices: international validation. J Rheumatol. 1994;21(8):1468-71.

21. Zhang C, Liang MY, Xu X, Zhang XW, Chen S. Clinical features of new-onset systemic lupus erythematosus in pregnant patients. J Obstet Gynaecol Res. 2018;44 (2):234-240. DOI 10.1111/jog. 13520.

22. Ku M, Guo S, Shang W, Li Q, Zeng R, Han M, et al. Pregnancy Outcomes in Chinese Patients with Systemic Lupus Erythematosus (SLE): A Retrospective Study of 109 Pregnancies. PLoS One. 2016 Jul 21;11(7):e0159364. DOI 10.1371/journal.pone.0159364.

23. Clowse ME. Lupus activity in pregnancy. Rheum Dis Clin N Am. 2007;33:237-52. DOI 10.1016/j.rdc.2007.01.002.

24. Baer AN, Witter FR, Petri M. Lupus and pregnancy. Obstet Gynecol Surv. 2011 Oct;66(10):639-53. DOI 10.1097/OGX.0b013e318239e1ee.

25. Nares MA, Hernández JA, Estrada A, Mendoza A, Cerveral CI, Villalva LD, et al. Lupus eritematoso sistémico activo durante el embarazo. Curso clínico y pronóstico en una Unidad de Cuidados Intensivos. Perinatol. Reprod. Hum. 2012 Jun; 26(2):96-105.

26. Hendawy SF, Abdel-Mohsen D, Ebrahim SE, Ewais H, Moussa SH, Khattab DA, et al. Pregnancy related complications in patients with systemic lupus erythematosus, an Egyptian experience. Clin Med Insioghts Reprod Health. 2011;5:17-24. DOI 10.4137/CMRH.S6862.

27. Petri M. Prospective study of systemic lupus erythematosus pregnancies. Lupus. 2004;13(9):688-9. DOI 10.1191/0961203303lu2006oa

28. Frodlund M, DahIström Ö, Kastbom A, Skogh T, Sjöwal C. Associations between antinuclear antibody staining patterns and clinical features of systemic lupus erythematosus: analysis of a regional Swedish register. BMJ Open. 2013;3(10), e003608. DOI 10.1136/ bmjopen-2013-003608.

29. Phansenee S, Sekararithi R, Jatavan P, Tongsong T. Pregnancy outcomes among women with systemic lupu erythematosus: a retrospective cohort study from Thailand. Lupus. 2018 Jan;27(1):158-164. DOI 10.1177/0961203317721353.

30. Lateef A, Petri M. Managing lupus patients during pregnancy. Best practice \& research Clinical rheumatology. 2013;27(3):435-47. DOI 10.1016/j.berh.2013.07.005.

31. Koh JH, Ko HS, Kwok SK, Ju JH, Park SH. Hydroxychloroquine and pregnancy on lupus flares in Korean patients with systemic lupus erythematosus. Lupus. 2015. Feb;24(2):210-7. DOI 10.1177/0961203314555352.

32. Clowse ME, Magder L, Witter F, Petri M. Hydroxychloroquine in lupus pregnanc. Arthritis Rheum. 2006 Nov;54(11):3640-7. DOI 10.1002/art.22159.

33. Ruiz-Irastorza G, Ramos-Casals M, Brito-Zeron P, et al. Clinical efficacy and side effects of antimalarials in systemic lupus erythematosus: a systematic review. Ann Rheum Dis. 2010 Jan;69(1):20-8. DOI: 10.1136/ ard.2008.101766.

34. Madazli R, Yuksel MA, Oncul M, Imamoglu M, Yilmaz H. Obstetric outcomes and prognostic factors of lupus pregnancies. Arch Gynecol Obstet. 2014 Jan;289(1):49-53. DOI 10.1007/s00404-013-2935-4.

35. Eudy AM, Siega-Riz AM, Engel SM, Franceschini N, Howard AG, Clowse MEB, et al. Effect of pregnancy on disease flares in patients with systemic lupus erythematosus. Ann Rheum Dis. 2018 Feb 20;77(6): 855-60. DOI 10.1136/annrheumdis-2017-212535.

36. Carmona F, Font J, Cervera R, Muñoz F, Cararach V, Balasch J. Obstetrical outcome of pregnancy in patients with systemic Lupus erythematosus. A study of $60 \mathrm{ca}-$ ses. Eur J Obstet Gynecol Reprod Biol. 1999;83(2):13742. DOI 10.1016/S0301-2115(98)00312-1.

37. Lockshin MD, Reinitz E, Druzin ML, Murrman M, Estes D. Lupus pregnancy: case-control study demonstrating absence of lupus exacerbation during or after pregnancy. Am J Med. 1984;77(5):893-8.

38. Lockshin MD. Preonancy does not cause systemic lupus erythematosus to worsen. Arthritis Rheum. 1989;32(6):665-70.

39. Al Arfaj AS, Khalil N. Pregnancy outcome in 396 pregnancies in patients with SLE in Saudi Arabia. Lupus. 2010 Dec;19(14):1665-73. DOI: 10.1177/0961203310378669.

40. Petri M. Prospective study of systemic lupus erythematosus pregnancies. Lupus. 2004;13(9):688-689. DOI 10.1191/0961203303lu2006oa. 
41. Clark CA, Spitzer KA, Nadler JN, Laskin CA. Preterm deliveries in women with systemic lupus erythematosus. J Rheumatol. 2003 Oct;30(10):2127-32.

42. Stojan G, Baer AN. Flares of systemic lupus erythematosus during pregnancy and the puerperium: prevention, diagnosis and management. Expert Rev Clin Immunol. 2012 JuI;8(5):439-53. DOI 10.1586/eci.12.36.

43. Moroni G, Ponticelli C. Pregnancy in women with systemic lupus erythematosus (SLE). Eur J Intern Med. 2016 Jul;32:7-12. DOI: 10.1016/j.ejim.2016.04.005.

44. Chakravarty EF, Colón I, Langen ES, Nix DA, El-Sayed $\mathrm{YY}$, Genovese MC, et al. Factors that predict prematurity and preeclampsia in pregnancies that are complicated by systemic lupus erythematosus. Am J Obstet Gynecol. 2005;192(6):1897-904. DOI 10.1016/j.ajog.2005.02.063.

45. Clowse ME, Magder LS, Witter F, Petri M. Early risk factors for pregnancy loss in lupus. Obstet Gynecol. 2006;107(2 Pt 1):293-9. DOI 10.1097/01. AOG.0000194205.95870.86.

46. Lazzaroni MG, Dall’Ara F, Fredi M, Nalli C, Reggia R, Lojacono A et al. A comprehensive review of the clinical approach to pregnancy and systemic lupus erythematosus. J Autoimmun. 2016 Nov;74:106-117. DOI: 10.1016/j.jaut.2016.06.016. 